\title{
An Analysis of Island Models in Evolutionary Computation
}

\author{
Zbigniew Skolicki \\ Department of Computer Science \\ George Mason University \\ 4400 University Drive, MSN 4A5 \\ Fairfax, VA USA \\ zskolick@gmu.edu
}

\begin{abstract}
A need for solving more and more complex problems drives the Evolutionary Computation community towards advanced models of Evolutionary Algorithms. One such model is the island model which, although the subject of a variety of studies, still needs additional fundamental research. In my Ph.D. thesis I am aiming at studying the behavior of island models with regard to the amount of cooperation between islands, the level of heterogeneity and the difficulty of the problem being solved. This paper presents the main ideas and gathers preliminary results.
\end{abstract}

Categories and Subject Descriptors: I.2.8 [Artificial Intelligence]: Problem Solving, Control Methods, and Search - heuristic methods; I.2.11 [Artificial Intelligence]: Distributed Artificial Intelligence; G.1.6 [Numerical Analysis]: Optimization - global optimization

General Terms: Algorithms, Performance, Experimentation

Keywords: evolutionary computation, island model, parameters, test suite, heterogeneity

\section{Keywords}

\section{INTRODUCTION}

To speed up an evolutionary computation it is a common practice to use multiple machines. Therefore we need to understand how to run evolutionary algorithm (EA) on many computers, what changes need to be done and how it affects the behavior of the system. Existing hardware solutions support the usage of island models (IMs), in which separate populations can reside on different machines, and an arbitrary topology of communication can be maintained using a computer network. Clusters of machines show even higher hardware integration and are another platform for running IMs. On the other side, grids, which are heterogeneous networks of loosely connected computers can be also used for computational purposes.

Permission to make digital or hard copies of all or part of this work for personal or classroom use is granted without fee provided that copies are not made or distributed for profit or commercial advantage and that copies bear this notice and the full citation on the first page. To copy otherwise, to republish, to post on servers or to redistribute to lists, requires prior specific permission and/or a fee.

GECCO'05, June 25-29, 2005, Washington, DC, USA

Copyright 2005 ACM 1-59593-010-8/05/0006 ...\$5.00.
IMs behave qualitatively different from standard EAs and even using them on a single machine may produce different, possibly better solutions for many problems, especially complex ones (for example in engineering). Separating individuals spatially from each other results in slowing down the information flow between individuals, which may have both desired and undesired results. A slower information flow may stop temporarily best solution from dominating the population and allow different building blocks or solutions to be discovered and later confronted, which is important in the context of engineering design and creativity. On the other hand one can prevent successful mixing, which could otherwise lead to constructing a novel solution.

There is a need for a better understanding of properties of IMs. Despite the intuition and the existing research on particular properties, there is still no general theory describing the behavior of IMs and explaining how to setup and manage IMs. Understanding the relations between parameters would be an important step towards this goal.

A diversification of islands further impacts the behavior of the model, and may intensify certain characteristics of the system, which is a reason for studying heterogeneous IMs. Particular subpopulations may be assigned to different goals. It is important to answer how heterogeneity is related to other parameters and when heterogeneous IMs are useful.

Using a model of how particular properties of an IM influence each other, it should be easier to construct adaptation mechanisms for IMs (e.g. based on the dynamics of search or self-adapting ones). Creating proper rules would diminish the number of parameters required to be set in IMs.

\section{BACKGROUND}

An IM is an approach to distribute EA. It divides individuals into subpopulations and allows for occasional exchange of individuals (migrations). The simplest island model assumes the same global parameters for islands and the same global parameters for migrations. Populations are characterized by their number, size and the EA type. Migrations are described by the topology (denoting which islands send individuals where - e.g. ring, torus or random topology), size, frequency, and the emigration/immigration policy (how we choose and insert migrants).

There has been much research done on IMs. Theoretical studies of takeover times were published for example by Rudolph [9]. The number and size of populations were studied by Whitley et al. [14]. Cantú-Paz studied various parameters of island models (among others the population size, the connectivity, the migration rate, the takeover time and 
the selection intensity), some of them on more theoretical models and others in more experimental setups [4]. There exist models where migrations are triggered when certain criteria are met (Branke et al. [3]), however fixed schedules are much more popular and easier to analyze. Fernandez et al. studied the population number and size, the topology and the migration size and frequency in the context of GP [6]. Alba and Troya studied the asynchronism of island models [1]. The diversity of populations was studied by Ursem [13] and Gustafson [7].

Different representations, operators, strategies, etc. within one system are possible in heterogeneous IMs, and they help overcome many artificial constraints imposed by choices regarding the solving method. Changes in the exploration distribution around a given solution due to different representations or genetic operators are described by neutrality theory [12]. An interesting application of a heterogeneous setup is Injection Island GA [5] or a related Hierarchical Fair Competition model [8]. Switching representations dynamically (by "shifting" Gray encodings) was presented in [2].

\section{RESEARCH DIRECTIONS}

The methodology used in the thesis will be primarily experimental. I will observe the changes in the behavior of an IM while changing its properties.

\subsection{Test suite and measurements}

An appropriate test suite needs to be created and proper measurements chosen. The test suite will consist of existing EA tests, modular functions, hierarchical problems and finally one or two engineering domains. Each test should have an adjustable complexity (e.g. by changing the number of dimensions). From existing EA test functions I will choose several that are multimodal and relatively difficult (e.g. Rosenbrock, Schwefel, Rastrigin or Griewangk functions). Modular problems should require that a recombination is used to solve them. For hierarchical problems for example Watson's H-IFF function should be a good choice. A test-case generator for realistic non-stationary landscape may be used [13]. A water distribution network simulator is another possibility due to some already existing computational framework and research.

Several measurements should be considered. The most obvious one is of course the fitness (specifically the best-sofar value). Others include the diversity of populations (local and global) and some measure of the migrants behavior (e.g. the number of their offsprings surviving) or others showing the inside dynamics of an EA.

\subsection{Relation between parameters}

Experiments with the migration size, the migration frequency, the migration policy (its strength in terms of selection pressure), the topology (connectivity/diameter) and the EA selection pressure should reveal their mutual relationship. For each pair of parameters, all combinations of their values will be systematically analyzed and the performance measured. The goal is to understand the driving forces of an IM.

Frequent and big migrations, strong migration policy, weak selection in islands and dense topology should all increase the importance of interaction between islands, relatively to the evolution inside islands. This should lead to sharing the same gene pool and ,intuitively, the behavior of such IM should resemble the behavior of a panmictic model. On the other hand, opposite values of the above parameters and the dominance of the evolutionary processes over the migrations yields a model resembling separated islands or multiple independent runs. For complex problems both cooperation and individual evolution is needed and therefore the right balance in terms of parameters is a key to achieve better results. The situation resembles striving for the right balance between exploration and exploitation.

Many parameters of IMs can be combined together to describe how much islands are independent in their evolution, and how much they cooperate with each other. It would be desirable to construct a single characteristic describing how much cooperation there is and show if and when the mapping Parameters $\rightarrow$ Cooperation $\rightarrow$ Performance can be used. It would reduce the dimensionality of an IM description and allowed for higher level studies with other properties.

It seems natural to assume that with a growing problem complexity, one will need more cooperation between subpopulations to solve the problem. In the same time, each population will have a more difficult and specific task to achieve and therefore it will need more and more independence. So, the problem should be solvable if there is not too much and not too little of cooperation. My assumption is that as the problem complexity grows, the limits come closer to each other, and finally for some complexity it becomes impossible to solve the problem.

\subsection{Heterogeneity in island models}

It seems that the evolution inside an island is more responsible for local changes, whereas migrations exchange already optimized solutions "grown up" in different environments and thus a better general structure can be obtained. Therefore island models should enable evolution at different (at least two) levels of generality. It also seems that the ability to explore various higher level structures of solutions depends on how independently and individually evolution proceeds in subpopulations. In addition, it is known that for example fluctuating the selection pressure, or sporadically increasing the mutation level are in general good ways out of a stagnation.

The restricted flow of information together with mating and selection limited to each island create natural irregularities and borders, which may function as seeds for randomly differentiating the evolutionary process and making it non-uniform. Differentiating islands (making the model heterogeneous) directly enhances the desired features of IMs, instead or counting on evolution getting diversified due to a stochastic process.

Different operators or operators' probabilities may influence the evolvability and regulate the degree and the direction of search. Different representations can in addition introduce "shortcuts" between distant solutions. Different fitness functions may "pull" evolution toward different goals and be used in a multi-objective problem; or may focus on different subparts of solutions, enhancing evolution by using a task division approach. Heterogeneity may also be achieved by changing standard parameters of EAs. One of the simplest options is to vary the selection pressure.

Since the heterogeneity causes a more independent search, it may be the case, that it should be balanced by an in- 


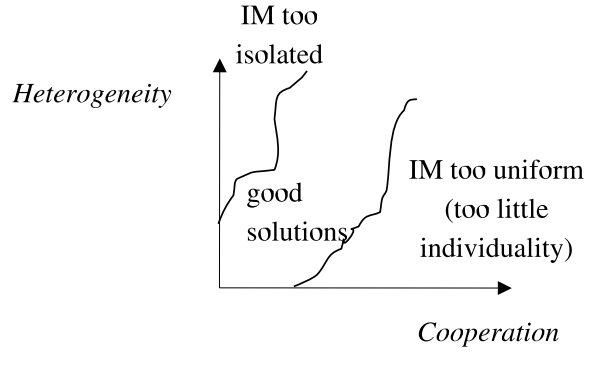

Figure 1: A hypothetical relation between the cooperation and the heterogeneity level

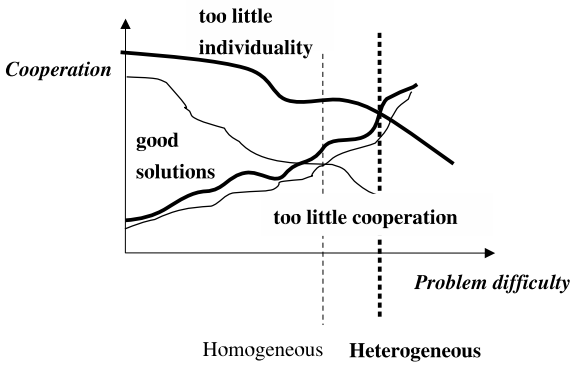

Figure 2: A hypothetical influence of heterogeneity on the solving capability of an IM

creased level of cooperation. I expect results similar to the hypothetical one shown in Figure 1. Heterogeneity should both increase the maximal allowed and the minimal required level of cooperation. Populations could cooperate more without the risk of becoming too similar, however they would also get isolated easier and evolve incomparable solutions. These changes could affect the solving capability of the IM, as shown in Figure 2.

By experimenting with the level of heterogeneity and previous parameters, I hope to answer how they are related, and when heterogeneity is useful. Both previous test functions and tests specially designed for heterogeneous islands will be used. The ultimate goal of this research will be to understand how cooperation level, the heterogeneity level and the problem difficulty influence the performance of an IM and how to choose the first two, given a specific problem to solve.

\subsection{Adaptation}

The part of research on adaptation will depend on the results from the previous parts. Rules for adjusting IMs should be created. As an example, one demanding bigger heterogeneity in the beginning and smaller towards the end of run may be created.

\section{PRELIMINARY RESULTS}

\subsection{Test suite}

Several functions were created in so-far experiments. Four of them were created with standard IMs in mind. Their aims were to study the negative influence of cooperation between islands (IM1), the positive influence of cooperation (IM2, IM3) and the influence of cooperation on overfitting

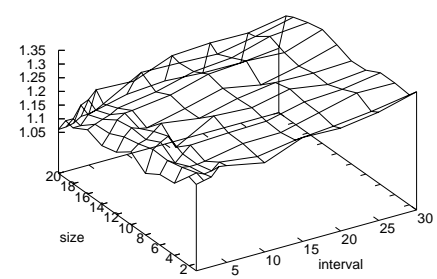

Figure 3: IM3, average best-so-far value $($ panmictic $=1.184$, separated $=1.12)$

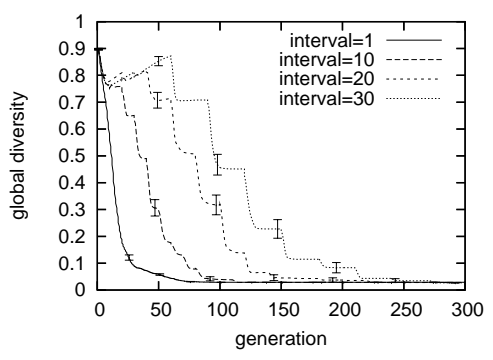

Figure 4: IM3, average global diversity in runs with different migration intervals, migration size $=\mathbf{1 0}$

to locally ideal solutions (IM4) [11].

Another function was designed for studying heterogeneous IMs $(F)$. The function is difficult for only binary or Gray encodings, but is much easier when switching between representation is allowed [10].

Additionally, standard multimodal functions, known as Rosenbrock, Rastrigin, Schwefel and Griewangk are also used.

\subsection{Migration sizes and intervals}

Two very basic and important parameters of island models are the migration size and interval. For different fixed settings we studied combinations of these two parameters within certain ranges. The results are presented in [11]. We experimented with setups of 2 and 5 islands, random (dynamic) topology and a policy choosing and replacing random individuals. The performance of the EA was measured with the average best-so-far value from all islands and multiple runs. IM1, IM2, IM3, IM4, Rosenbrock, Schwefel, Rastrigin and Griewangk functions were used. An exemplary result for IM3 function is shown in Fig. 3. Additional analysis of diversity helped explaining the behavior of the IM.

Very frequent migrations turned out disadvantageous. Measuring genetic diversity showed that too frequent migrations cause islands to dominate others and lose global diversity before they are able to exchange solutions to produce better results. If one waits long enough before migrating, the global diversity remain large, even though local diversities drop between migrations (Fig. 4). This means that different islands converge to different peaks. Exchange of individuals at this point results in a better average fitness.

Rare migrations cause the model to converge slower. Therefore if the time is limited, too large intervals should be avoided. Interestingly, with large intervals, moderate size migrations caused the fastest convergence. Large sizes probably prevented mixing of populations and small migrations 


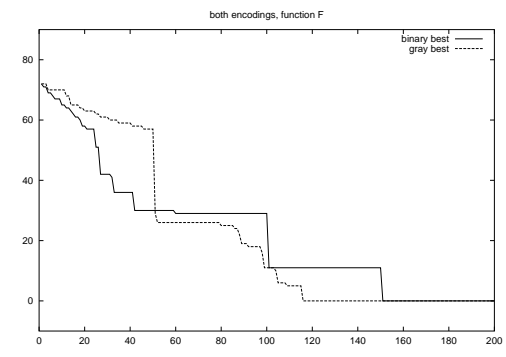

Figure 5: A single run of a heterogeneous IM. The mutual influence of populations by means of migrations is very visible

happened to boost diversity. We observed little sensitivity of best-so-far value to the migration size of small and moderate values, whereas migrating whole populations obviously degraded the performance. Apparently, even a small migration is enough to maintain cooperation between islands.

In general, we found that the performance was determined by at least several factors and thus one cannot base it simply on the level of cooperation. However, for moderate values of the parameters we could notice a similarity in the behavior of runs with the same average number of individuals per generation, so maybe within certain ranges we could combine parameters into fewer ones. The issue needs more investigation.

Another side result from the experiments is that the island model can produce better results and is truly qualitatively different from both the panmictic and separated populations models. IM's "sweet spot" contained small to moderate values of parameters and is not connected to regions that would correspond to one big population (frequent big migrations), nor to many separated ones (rare and very small migrations).

\subsection{Heterogeneous representations}

In another set of experiments with IMs we used different representations in each island [10]. The model transforms individuals from one representation to another during migrations. A local optimum in one representation need not be a local optimum in the other representation. Therefore by switching representations in some cases we are able to "escape" from such suboptimal solutions and to solve problems that are difficult for single representation EAs.

We illustrated this approach with a two population island model in which one island uses a standard binary encoding and the other island uses a standard reflective Gray code. The function $F$ from the test suite was used. Not surprisingly, single populations using just one encoding usually fail to find the global optimum. The situation changes when we allow for the island model with both representations in the same time. On Figure 5 the best-so-far curves from a single run with two representations and rare migrations (every 50 generations) are shown. One can clearly see the influence of migrations and the switching roles of populations which help each other.

To study the robustness of the model we conducted several sets of experiments. We tested the model using two crossover types (two-point and uniform) and two selection strategies (ranked with elitism and binary tournament). Similar analysis was performed for different levels of migration.
Finally the behavior on Rosenbrock, Schwefel, Rastrigin and Griewangk functions was tested. The IM was found either comparable or statistically better than single representation EAs on the tested problems.

\section{CONCLUSIONS}

The preliminary experiments mostly confirm our predictions about IMs. Some are however counter-intuitive. It is important to understand whether these are exceptions from the theory, or whether we should update the model. To understand what the reasons for such unexpected behaviors are, we need to know what happens inside the islands. This can be achieved by using proper measures (for example tracking different genes through generations). Of course, further experiments are needed.

\section{REFERENCES}

[1] E. Alba and J. M. Troya. An analysis of synchronous and asynchronous parallel distributed genetic algorithms with structured and panmictic islands. In IPPS/SPDP Workshops, pages 248-256, 1999.

[2] L. Barbulescu, J.-P. Watson, and D. Whitley. Dynamic representations and escaping local optima: Improving genetic algorithms and local search. In $A A A I / I A A I$, pages 879-884, 2000.

[3] J. Branke, A. Kamper, and H. Schmeck. Distribution of evolutionary algorithms in heterogeneous networks. In Proceedings of Genetic and Evolutionary Computation Conference - GECCO 2004. Springer-Verlag, 2004.

[4] E. Cantú-Paz. Efficient and Accurate Parallel Genetic Algorithms. Kluwer Academic Publishers, 2000.

[5] D. Eby, R. Averill, E. Goodman, and W. Punch. The optimization of flywheels using an injection island genetic algorithm. In P. Bentley, editor, Evolutionary Design by Computers, pages 167-190. Morgan Kaufmann, San Francisco, 1999.

[6] F. Fernández, M. Tomassini, and L. Vanneschi. An empirical study of multipopulation genetic programming. Genetic Programming and Evolvable Machines, 4(1):21-51, March 2003.

[7] S. M. Gustafson. An Analysis of Diversity in Genetic Programming. PhD thesis, The University of Nottingham, 2004.

[8] J. Hu and E. Goodman. The hierarchical fair competition model for parallel evolutionary algorithms. In Congress of Evolutionary Computation, CEC 2002, IEEE World Congress on Computational Intelligence, 2002.

[9] G. Rudolph. On takeover times in spatially structured populations: Array and ring. In K. K. Lai, O. Katai, M. Gen, and B. Lin, editors, Proceedings of theSecondAsia-Pacific Conference on Genetic Algorithms and Applications(APGA '00), pages 144-151, Hong Kong, PR China, 2000. Global-Link Publishing Company.

[10] Z. Skolicki and K. De Jong. Improving evolutionary algorithms with multi-representation island models. In Parallel Problem Solving from Nature - PPSN VIII 8th International Conference. Springer-Verlag, 2004.

[11] Z. Skolicki and K. De Jong. The influence of migration sizes and intervals on island models. In Proceedings of the Genetic and Evolutionary Computation Conference (GECCO-2005). ACM Press, 2005.

[12] M. Toussaint and C. Igel. Neutrality: A necessity for self-adaptation. In Proceedings of the IEEE Congress on Evolutionary Computation (CEC 2002), pages 1354-1359, 2002.

[13] R. K. Ursem. Models for Evolutionary Algorithms and Their Applications in System Identification and Control Optimization. PhD thesis, University of Aarhus, EVALife, Dept. of Computer Science, Denmark, 2003.

[14] D. Whitley, S. Rana, and R. B. Heckendorn. The island model genetic algorithm: On separability, population size and convergence. Journal of Computing and Information Technology, 7(1):33-47, 1999. 